\title{
Expecting the Unexpected: Safety Considerations for Ethnobotany Field Research
}

\author{
K. W. Bridges and Will McClatchey
}

\section{Research Methods}

\begin{abstract}
All ethnobotanical field researchers should be prepared for emergencies. While such problems are often thought to be low probability events, they are actually common enough that all investigators should be appropriately prepared every time they go into the field, even in areas that are nearby and familiar. Preparation involves a series to steps that should be started early, well before going into the field. The first part of the planning involves thinking about the area, intended activities and the potential for danger. This is followed by assembling materials and procedures that will be helpful in the most likely dangerous situations. It is also important to know, ahead of time, what to do in a broad range of emergencies. Every field researcher should have good safety kits, be trained in their use, and practice sufficiently so that safety procedures become a habit.
\end{abstract}

\section{Introduction}

We didn't expect to get into trouble. We were simply traveling between two nearby Pacific atolls in a boat that was sufficiently large and run by an experienced crew. After several hours at sea we realized that we should have already reached our destination. This prompted us to check our GPS. Fortuitously, we had previously recorded a waypoint close to our destination. What we discovered was that we were traveling about 90 degrees off course. Without an abrupt course change we would soon be far out to sea, out of gas and, in this particular region, unlikely to be rescued. Without a rescue, we would have died.

We did make it to our destination safely due to our always carrying a GPS, making sure to have ample charged batteries, and frequently recording waypoints. Note that all three habits were needed.
On another trip, we were working in a very familiar area when five people in the party were unexpectedly stung by wasps. Two of these people experienced rapid allergic reactions. This was a dangerous situation as we were several hours from medical assistance. Fortunately, we were carrying antihistamine medication and quickly reduced the anaphylactic reaction to a manageable condition.

These were two of many experiences that we have had conducting field research. When we shared our concern for safety with our colleagues, we found that our encounters with the "unexpected" weren't at all rare. All of these seasoned field researchers had several stories about misadventures (Table 1). Some even told us of near death and deadly experiences. Fortunately, as in our case, all of these threatening situations turned out favorably.

We have drawn several conclusions from these experiences.

\section{Correspondence}

Kim Bridges, Department of Botany, 3190 Maile Way, 101, University of Hawai'i at Manoa, Honolulu, Hawai'i 96822. U.S.A. kim.bridges@gmail.com

Will C. McClatchey, Department of Botany, 3190 Maile Way, 101, University of Hawai'i at Manoa, Honolulu, Hawai'i 96822. U.S.A.

mcclatch@hawaii.edu

Ethnobotany Research \& Applications 4:041-050 (2006) 
Table 1. Unexpected events related to us in a small sample of professional field scientists. Several of these incidents were reported more than once.

\begin{tabular}{|c|c|}
\hline $\begin{array}{ll}\text { Medical problems } \\
\text { - } & \text { Broken leg } \\
\text { - } & \text { Chemical burn from plant sap } \\
\text { - } & \text { Cuts } \\
\text { - } & \text { Dehydration } \\
\text { - } & \text { Dermatitis } \\
\text { - } & \text { Fye infection hook through finger } \\
\text { - } & \text { Insect stings } \\
\text { - } & \text { Kidney stone } \\
\text { - } & \text { Large infected wound } \\
\text { - } & \text { Major sunburn } \\
\text { - } & \text { Malaria } \\
\text { - } & \text { Migraine headache } \\
\text { - } & \text { Snake bite } \\
\text { - } & \text { Toothache }\end{array}$ & $\begin{array}{l}\text { Transportation Equipment Problems } \\
\text { Boat } \\
\text { - Capsized in ocean } \\
\text { - } \quad \text { Capsized in river } \\
\text { - } \quad \text { Engine failure in dangerous surf } \\
\text { Horse } \\
\text { - Injured } \\
\text { - } \quad \text { Ran away } \\
\text { Truck } \\
\text { - } \quad \text { Keys locked in truck in isolated forest } \\
\text { - } \quad \text { Overturned in steep terrain } \\
\text { - } \quad \text { Stuck in mud } \\
\text { Walking } \\
\text { - } \quad \text { Damaged boot sole } \\
\text { - } \quad \text { Blisters } \\
\text { Other Transport } \\
\text { - } \quad \text { Public transport cancelled }\end{array}$ \\
\hline $\begin{array}{ll}\text { General Equipment Problems } \\
\text { - } & \text { Broken } \\
\text { - } & \text { Dead batteries } \\
\text { - } & \text { Stolen } \\
\text { - } & \text { Wet so will not work } \\
\text { - } & \text { Forgot instructions }\end{array}$ & $\begin{array}{l}\text { Other } \\
\text { - } \quad \text { Hysterical field assistant } \\
\text { - } \quad \text { Lost in a forest (spent the night in the rain) } \\
\text { - }\end{array}$ \\
\hline
\end{tabular}

- $\quad$ Significant problems are not unusual while doing field work.

- $\quad$ Experience is unlikely to be sufficient to avoid serious problems.

- $\quad$ Being prepared is important in all situations, not just those where you think you might be endangered.

- Safety involves a combination of advance planning, knowledge, equipment and supplies, and practice.

There is obviously no magic kit that will keep you safe given the variety of situations that may be encountered. However, there are some plans that should be made, procedures that can be learned, and information and supplies that can be carried that may help. If you are an active field researcher, sooner or later you will encounter an emergency situation. Your preparation for that moment will be critical in determining the outcome.

Safety concerns range from individual problems, such as spraining an ankle, to those that impact large populations such as hurricanes, earthquakes, landslides, volcanic eruptions, floods and tsunami. Large-scale, natural disasters cause tens of thousands of deaths and hundreds of thousands of injuries every year (Dilley et al. 2005). Other problems may be the result of human activities, such as a violent attack, or even mechanical breakdowns. Preparedness should consider the full range of possibilities, from those resulting from your own activities to those caused by events that impact the safety of many people.
We are not going to discuss the need for preparation regarding water, food, clothing, or shelter except in an emergency context. Meeting basic needs is important but outside the scope this paper.

Fortunately, there is a wide set of literature that examines kinds of dangerous situations that are likely to occur when working under field conditions (Brown 1983, Tawrell 1996). Field workers should consider the following categories of concern:

- Physical trauma

- Infections

- Environmental exposure

- "Way finding"

- Loss of essential equipment

- Loss of basic needs (e.g., potable water, food, clothes, shelter)

For each of these categories, there is knowledge, techniques or skills, and materials (equipment and supplies) that are appropriate to address these concerns. Although many materials may be acquired from the environment, we will focus primarily on those items that can be brought along.

The following discussion makes several assumptions, although many of the implications go beyond these limitations. First, our focus is on field researchers working in areas where they did not grow up or where they have not 


\section{Bridges and McClatchey - Expecting the Unexpected: Safety Considerations for Ethnobotany Field Research}

developed survival skills. We assume that no help is available in the local community or that it is unreliable. Our concerns are for situations where environmental materials are only minimally available for foods, shelter, fuel and medicine or where these materials may be unknown. Also, we assume that survival for only one or two days is need before assistance is available.

It is important to note that there are insurance programs available that will provide evacuation assistance in extreme situations. The Divers Alert Network (www.diversalertnetwork.org) program for SCUBA divers is a model of such insurance. This network can even provide emergency evacuation for non-divers. Also, most major academic institutions offer courses in field safety and may require safety training for field researchers, including students (e.g., www.stanford.edu/dept/undergrad/urp/PreField/).

\section{Expect the Unexpected}

An ounce of prevention is worth a pound of cure. Benjamin Franklin

Safety preparedness involves systems thinking (Senge 1990). This means that you need to lay out all aspects of the situation and outline the safe versus unsafe actions. This includes what might happen to you, how you would respond, and what is needed to help you respond. Be sure to include identifying any ways to avoid problems in the first place. Once you have outlined the attributes of the system, each attribute needs to be evaluated. In the end, you should have a set of practices, lists of equipment and supplies, and training needs.

Building a system view starts well before you leave for any expedition (even if you are traveling a short distance to a well known field site). The first step is to fully assess the situations that you expect to encounter; Piven and Borgenicht (1999) list extreme examples just in case you're having problems getting your imagination started. Some problems are generic, such as minor health concerns that can be helped by over-the-counter medications. Power outages, inclement weather, and insect pests fit this category of routine likely occurrences. Other problems, such as getting lost and becoming seriously injured are much less likely but potentially life threatening.

This first stage of planning is additive. The goal is to be as comprehensive as possible.

- Consider the specific locality of the research. You should find out about the expected range of environmental conditions and the likelihood of unusual events. You should also know about the infrastructure of the area, including transportation, housing, and health care. This evaluation should include the water and food supply.

- Tabulate the expected activities and whether you will be doing them alone or with other people. Anticipate the experience and preparedness of any people who will be with you.

- List all potential emergency situations for which some intervention is useful or essential.

- Assess what actions you would take for each situation.

- Determine what knowledge and skills would be helpful in all of the situations.

- Determine what materials (supplies and equipment) would be helpful in all of the situations.

- $\quad$ Sketch the procedures you need to use that combine knowledge and skills with materials in order to handle the situation. For example, if you are lost, you should know how to compare your current location with a stored waypoint (knowledge), be trained to load waypoints of key locations (skill), and carry a fully functional GPS (material).

The next stage in your planning involves evaluation. The first stage has probably resulted in specifications for more contingencies than you're likely to be able to accommodate. You have probably listed more skills than you are ready to learn and your equipment list is likely to have too much "stuff." You need a realistic reevaluation of your needs. Look at the situation with the following perspectives.

- What are the probabilities of occurrence versus the negative implication of each type of situation? Are some contingencies so unlikely that they can be addressed through avoidance of certain situations? For example, if you do not have equipment or skills to address different kinds of water accidents or injuries, then it may be best to avoid work involving water hazards.

- What quantities of supplies are appropriate? Perhaps you don't need a full bottle of a medicine if a few pills will be adequate.

- $\quad$ Can substitutions cover multiple needs? Is it possible to choose equipment that can run with the same type of battery in order to avoid carrying several kinds for emergencies?

- $\quad$ Can learning one skill address several different kinds of emergency situations? For example, learning to tie 
a few kinds of knots can be useful in addressing a number of different situations.

In the end you should have concrete ideas on the key knowledge, skills and materials needed, within the limits of your resources, to promote your safety. Together this should provide confidence, as well as a sense of what can happen, that will help you avoid emergencies. Hopefully you will never need to use your knowledge, skills and materials other than for practice.

\section{Safety Procedures}

What do you do if you are in the field and something goes wrong? Thinking about this before you encounter a difficult situation can have several benefits, the most important of which is that you do not see this as an emergency.

For instance, the ability to start a fire in a wet environment can be the difference between life and death. For those of us who have the knowledge, skill, and materials to make a fire, we simply make one and do not consider that we were in an emergency situation. Alternatively, those who do not have the knowledge, skill, or materials to make a fire will find themselves in an emergency situation. Note that even in this simple example all three elements are needed to differentiate an emergency situation from just another day of field research.

Some safety situations are likely to be those you've encountered before and handled successfully. You probably don't need to be concerned with those. New situations will likely involve some research. Consider the following situations:

- You find yourself alone and off a trail?

- You find yourself floating in a river after your canoe has capsized?

- Your faithful field guide, who is the only one who knows where to go, is unconscious from a fall and cannot guide either of you to safety?

- $\quad$ You are stung on the face by bees and cannot see to look through your safety kit?

- $\quad$ Your team leader is suffering from malaria and cannot be moved from a remote location until she recovers?

- A member of your field team has become panicked with fear?

- $\quad$ Your dry pack with GPS and all other equipment falls into a lake and sinks to the bottom?

- $\quad$ You are working alone and twist your leg and need to contact someone for help?
Some planning is best done before you leave for your field trip. Discovering the "best practices" advice can be a straight-forward task if you have resources. For example, you can search the rich resources of the world-wide web for information. You are also more likely to be thoughtful in a relaxed situation that doesn't demand quick action.

\section{Medical Kits versus Safety Kits}

Medical kits designed for field use are generally limited to health-related situations. They contain items that you would find in a typical first-aid kit and are generally supplemented with a few items typically needed for a hiker (moleskin, elastic bandage) and outdoor injuries (sting relief, sunburn relief). Most of the over the counter medical kits that we have examined in stores are either worthless or worse because they contain poor quality supplies, in poor quantities, lack instructions for use, and are generally more bulky or fragile than they are worth. If your life will depend on a medical kit, get one that will save it.

Safety kits, as they are discussed here, are a superset of the medical kit. All of the medical kit items are included along with those supplies, tools and information that will help you find your way, stay dry and warm, signal other people, build a fire, collect and purify water and do minor equipment repairs. In the case of the vehicle kit, you should also be able to do minor repairs and solve some problems that might otherwise leave you stranded (such as clearing a tree blocking the road).

The overall design of a safety kit is highly dependent on the place it will be used (e.g., coastal, high mountain, temperate, tropical, desert), how far away you will be from assistance, how many people are involved, and the general skill-level of the group.

\section{Assembling Safety Kits: The Three Level Strategy}

We propose that you design and use three safety kits. The field kit will be carried with you all the time. The vehicle kit (for a car, truck, boat or plane) has two parts: one is for vehicle repairs and the other contains emergency supplies that supplement your field kit. The objective is that your field kit should be adequate to get you back to your vehicle. Then the vehicle supplies can be use to continue or enhance the treatment. The base camp kit is designed to handle the needs of a group. It has a greater breadth of treatment than the other two kits.

We present some of our ideas for contents in Appendices A-C. These are suggestions that are influenced by the places we typically work (tropical and subtropical environments, no more than 12 hours from some assistance, working with fairly well-trained companions from local communities). Examine world-wide web resources for information and sources of supplies. In particular, use search terms such as "ultralite survival kit" or "ultralite 


\section{Bridges and McClatchey - Expecting the Unexpected: Safety Considerations for Ethnobotany Field Research}

emergency kit" to find some pretty remarkable web pages describing and reviewing different mixes of supplies and equipment.

At this stage, you will likely want to start trying your ideas using real supplies and equipment.

A cheap way to practice is to determine what is needed and then cut out pieces of paper or cardboard that are about the correct size as the items for a particular kit. Write the name of the item on the paper and then use these to visualize the bulk of possible materials before buying them. Likewise, prepare pieces of paper for skills, knowledge, and equipment with the knowledge morphing into sets of instructions and the skills becoming checklists of things to practice before going on a field trip.

We segregate items that are intended for regular use or consumption from those that are stored in a safety kit. Daily food and water rations are considered separately from emergency supplies and are not packaged with safety supplies and equipment. Also, working tools, such as a GPS, are kept outside the safety kit (unless, of course, a separate GPS is stored as an emergency backup). One of the most commonly recommended items used in emergency situations is a knife. However, many field researchers carry a knife as part of their regular equipment. If a researcher knows how to use a knife effectively, it may be wise to include an emergency knife within the safety kit as backup for loss of this very important tool.

The safety kits carry materials that will be useful in an emergency. If you don't have the safety kit when it is needed, it is obviously worthless. One of the keys to actually taking a safety kit along is its packaging. In addition, the materials must be protected. If you leave a safety kit behind because it is too big or heavy, or if it is pilfered, or the contents are ruined because they were crushed, it will obviously have little or no benefit.

The field kit might contain key emergency items such as materials to start a fire, an emergency flashlight, medicines for headaches and anaphylaxis, small bandages for cuts, materials to start a fire, and a compass. The goal is to have it be as small as a deck or two of playing cards. The base-camp kit is larger, but still small enough that you could carry it in your pack on more distant trips. The containers for the two types of kits should be as small as possible. Choose durable materials that have a watertight seal. The vehicle kit need not be as small or easy to carry. It should be very durable, watertight, and fit the storage constraints and abuse associated with the specific vehicle.

A base camp kit might contain key items that are useful for a team but are too bulky to carry on a daily basis. Examples include larger bandages and materials for setting broken bones, fishing equipment or other emergency means of obtaining food and fresh water, survival tents that are carried in addition to normal camping materials and only used in extreme weather, back-up supplies for starting fires that can be used when other means fail, and other backup equipment and supplies (spare batteries, flashlights, emergency transponder, weather radio, strobe light, etc.)

A vehicle kit might include tools needed to repair the vehicle, a repair manual, emergency food and water supplies or materials suitable for getting food and water from expected environments, shelter materials that can be used to improve the shelter quality of the vehicle, equipment and supplies that can be used with parts of the vehicle to meet needs in an emergency. For example steel wool can be used with the vehicle battery as a means of starting a fire.

We keep these kits closed unless there is a need for their use. You should inspect each kit before each trip, replacing batteries and other expendables as needed.

\section{Important Paperwork}

Each safety kit must have a complete inventory list. This is kept on the top so that it is not necessary to sort through the materials to find out what is in the kit. Consider that although you might remember what is in the kit, you might be depending on someone else to use the kit. Copies of the kit contents should be distributed to the others on the trip before going to the field. This will encourage their own kit development and allow them to know that you have a kit if something happens to you.

We also include instructions for using the kit's contents. Documentation covering the medicines includes both the indications as well as the dosages. The information sheets are laminated so that they remain legible and are easily found.

\section{Training and Habits}

How long has it been since you have tried to start a fire under field conditions? Perhaps it's time to refresh your skills. Not only will this provide confidence but it will test your choice in equipment. Several of our students thought they could start a fire with a tiny magnifying glass. Their failure to sufficiently heat materials caused them to reconsider this fire starting strategy. The important point is that it is much better to discover shortcomings such as this before the procedures are actually needed.

Habits are, in many ways, the most critical part of the overall preparedness system. Good habits prevent problems and mitigate their impacts when they do happen. There are some procedures that you should make part of all of your field activities. Here are a few ideas (a more com- 
plete listing of suggested procedures is provided in Appendix D):

- Ask about local conditions. What are the potential dangers from sudden weather changes, poisonous plants or wild animals? Gather and heed advice from people who are experienced in the area.

- Make a list of the names of key people with whom you are working and carry this with you. This should include contact information, if possible.

- Record as GPS waypoints and appropriately label the locations of important places. This should include places like villages, road intersections, trail-heads, river crossings, hotels, field stations, airports, fences and other features that can assist in orientation.

- Determine the nearest locations of emergency services and how to make contact.

- Carry adequate money so that you can obtain needed services. This is related to the axiom for effective travelers, attributed to Sherwin Carlquist: "more money, less clothes."

\section{Discussion}

As ethnobotanists, we enjoy learning about the myriad ways in which people use the knowledge of plants and skills they have developed to survive and thrive in almost every environment on Earth. Daily challenges for people who grow up living in a particular environment (New York City, Baffin Island, Rongelap Atoll, Kilimanjaro, etc.) are met with a set of skills, material culture, and knowledge of how to deal with the situation. A normal person is able to deal with life in "normal" daily situations. However, when the situation is "abnormal" even a normal person may be faced with an emergency that could be a matter of life and death. In field research we typically find ourselves in an environment that is different from the one in which we grew up and know how to handle. Our concern is how to deal with abnormal situations in order to mitigate or eliminate potential harm, thus leading to a more satisfying and productive field research experience.

To a certain extent this paper is intended to modify situations from being abnormal (i.e., a person does not have knowledge, skills, and materials needed to cope) to a normal situation (i.e., a person has all of the knowledge, skills, and materials needed to cope). It is not an emergency when someone breaks their leg walking down the street in front of a hospital, has their car break down near a trusted service station, or suffers an allergic response to pollen and uses an inhaler or medication to self-remedy the situation. However, each of these situations could be an emergency if it happened in another context. Field safety is thus an issue of changing the context into one that is manageable, as "normal" as possible, and therefore not an emergency but just an inconvenience that is part of life.

You must always remember the context of your knowledge and experience. Although you might consider yourself an expert, you still could be at risk. This might be because of a new type of problem being studied or doing research in a new region.

Sometimes you will find yourself at risk because of the failure of some equipment. Prudent planning involves anticipating the need for some redundancy.

It is too easy to consider safety preparation as simply an exercise in carrying some equipment and supplies. While that is important, it is insufficient.

\section{Our recommendations:}

- Preparing for the unexpected is not a one-time activity. Each time your field work locations and situations change you should you re-evaluate your safety plans.

- Develop strong habits that make it natural to use your preparedness procedures. Practice these not just in extreme field situations, but wherever you are working in the field.

- Share preparedness information with colleagues. A team approach is not only more substantial, but it helps to reinforce the need for preparation among all members on the field trip.

- Be receptive to learning new techniques from people who are experienced in areas and environments that are new to you.

- Work collaboratively with local experts and they will take some responsibility to care for you.

- Strive to maintain a high level of preparedness while minimizing the bulk and weight of equipment and supplies that you carry.

\section{Literature Cited}

Brown, T., Jr. 1983. Tom Brown's Field Guide to Wilderness Survival. Berkely Books, New York, NY.

Dilley, M., R. S. Chen, U. Deichmann, A. L. Lerner-Lam, \& M. Arnold. 2005. Natural Disaster Hotspots: A Global Risk Analysis. World Bank Publications. Washington, D.C. 
Piven, J. \& D. Borgenicht. 1999. The Worst-Case Scenario Survival Handbook. Chronicle Books, San Francisco, CA.

Senge, P.M. 1990. The Fifth Discipline. Doubleday, New York, NY.

Tawrell, P. 1996. Camping \& Wilderness Survival: The UItimate Outdoors Book. Tawrell Publishers, Green Valley, Ontario. 
Appendix A. Example Field Kits.

This is not a generic field kit. What is included has been designed for the author's perceived needs working in Tropical Pacific Islands. It serves as an example that you should modify for the conditions and situations that you expect to encounter.

Kits with two purposes are detailed here. The ultralite kit is designed to fit in a pocket and always be carried while in the field. The hiking kit goes into a pack on day trips.

Three points are extremely important regarding the development of a field kit:

- It is important to be concerned with how to use everything you take. For example, we write down the conditions under which we should take a particular medicine, the dosage and the maximum duration of the treatment.

- $\quad$ Building your own kit is an active exercise in renewing your understanding of basic first aid, safety protocols, and emergency procedures. Telling you that you will be safe if you carry item $X$ has much less value than you doing your own research so that you have a kit filled with items that you understand and trust.

- $\quad$ Locate world-wide web resources that provide reliable basic health information. For example, the Merck Manual (www.merck.com) is a well-established source of information about an immense variety of medical concerns. Check their recommendations on appropriate basic treatments.

Kit 1. Ultralite Kit. The foundation of this kit comes from the Doug Ritter's Pocket Survival Pack produced by Adventure Medical Kits. Everything is held in a zip-opening plastic container $4.5 \times 5.5 \times 1$ in. Some items are organized in small plastic bags or containers.

\begin{tabular}{|l|l|l|}
\hline Fire starter & Duct tape & LED microlight \\
Tinder & Stainless steel utility wire $(6 \mathrm{ft})$ & Fishing kit (hooks, split shot, swivel) \\
Whistle & Braided nylon cord $(10 \mathrm{ft})$ & Pencil \& waterproof paper \\
Signal mirror & Heavy nylon thread $(50 \mathrm{ft})$ & Scalpel blade \\
Compass & Heavy-duty sewing needle & Fresnel lens magnifier \\
& Safety pins & Uncle Bill's tweezers \\
& Heavy aluminum foil $(3 \mathrm{ft} 2)$ & Wire saw (24") \\
& Antihistamine & Small locking single-blade folding knife \\
& Towelette (cleaning + fire tinder) & \\
\hline
\end{tabular}

Kit 2. Hiking Kit. The focus of these materials is on support for an unexpected overnight stay in the field. It is expected that the ultralite kit will be used in conjunction with the hiking kit. Cloth or plastic zippered container $5 \times 6.5$ $x 3$ in.

\begin{tabular}{|l|l|l|}
\hline Plastic poncho & Antihistamine & Facial tissue \\
Emergency blanket & Ibuprofen & 3 LED flashlight (80 hour) \\
(aluminum/plastic) & Large bandages & AAA batteries (3 ea for flashlight) \\
Bandana & Moleskin & Braided nylon rope \\
Insect repellent & Butterfly bandages & Single blade, folding locking 3" knife \\
Aloe sun block & Water purifying tablets & \\
\hline
\end{tabular}




\section{Bridges and McClatchey - Expecting the Unexpected: Safety Considerations for Ethnobotany Field Research}

Appendix B. Example Base Camp Kit

Base Camp Kit. This kit holds larger quantities of supplies so that field kits can be replenished. There are also some supplies that handle less-frequently encountered problems or those whose treatment can be delayed.

\begin{tabular}{|l|l|}
\hline $3 \times 3 "$ sterile gauze sponges & Pain medicines \\
$5 \times 9 "$ sterile ABD pads & Antihistamines \\
Cotton tipped applicators & Antiseptic creams and ointments \\
Sports tape/Elastic Bandage & Anti-diarrhea medicine \\
2" gauze bandage & Calamine lotion \\
3 "' x 3" adhesive bandages & Sunburn lotion \\
Triangle bandage & Sunburn relief \\
Towelettes & Instant cold pack \\
Alcohol prep pads & Instant heat pack \\
Moleskin & Sterile water \\
\hline
\end{tabular}

Emergency first-aid book Scissors

Mirror

Razor blade (or scalpel blade)

Large emergency knife/

saw with 6-10" blade

Appendix C. Example Vehicle Kit.

Part 1. Vehicle Repair. This kit assumes that the spare tire, jack and lug wrench (and any other tire changing equipment) is present and in good working order.

\begin{tabular}{|l|l|l|}
\hline Waterproof matches & Vehicle maintenance book & Folding shovel \\
Work gloves & Fan belt repair kit & Bow saw \\
Rain poncho & Jumper cables & Axe/Hatchet \\
Hand warmer & Tire inflator (can with sealant) & Large emergency knife/ \\
Towelettes & Tire pressure gauge & saw with 6-10" blade \\
Waterless hand cleaner & Screwdriver (flathead \& Phillips) & Small fire extinguisher \\
Shop cloth/Paper towels & Crescent wrench (small + medium) & \\
Flashlight \& extra batteries & Allen wrenches & Siphon \\
Strobe Light & Pliers & Funnel \\
Emergency light stick & Socket wrenches (3/8" & Gas can \\
Popup traffic cones/triangle & with extensions) & Tow rope (15') \\
Can opener & Vise grips & Spare change \\
Cable ties & Wire cutter & Prepaid calling card \& \\
Super glue & Multifunction knife & emergency numbers \\
Silicone sealing tape & Hammer & Emergency radio (receive weather info) \\
Duct tape (high-performance) & Fuses & Whistle \\
WD-40 lubricant & Replacement fluids (water, oil) & \\
\hline
\end{tabular}

Part 2. Vehicle-Based Emergency Supplies. These are used to supplement and extend the field kit supplies.

\begin{tabular}{|l|l|l|}
\hline Adhesive tape & Plastic bandage strips & Disinfectant \\
Sterilized gauze bandages & Pain relievers & Hand sanitizer \\
Sterilized gauze pads & Antihistamines & Small scissors \\
\hline
\end{tabular}


Appendix D. Ten Key Activities to Promote Field Safety.

The following list of activities assumes that you will be working at a field site (your destination) that is some distance from where you ordinarily live (your origin). None the less, all of the points should be considered, independent of the destination.

Note that many of these activities must be done before you depart. Most also require that you keep up to date as you work at your destination.

1. Understand the lay of the land. Explore the landscape of the area in which you will be working through the use of maps, particularly with resources such as Google Earth. Find maps that have useful information (such as vegetation cover) and overlay them on the destination area. Be aware of key landmarks such a major highways, rivers, mountains, railway lines and use these to keep track of where you are.

2. Compile waypoints and load them into a GPS. Develop the habit of recording the digital locations (latitude and longitude) of key places as you do research about an area. Look up locations of hospitals, transportation hubs and government centers. Add to this list (and your GPS) as you work at your destination.

3. Write down key names, addresses and contact information. This should include people and institutions from your origin (e.g., your doctor, dentist, bank, and people to be notified in case of emergency) as well as your destination (e.g., host colleagues, where you will be staying). Identify a trusted person at your destination. Keep this list up to date. Store a copy in a safe place and distribute copies of this information to those who receive copies of your travel emergency plan.

4. Develop a financial emergency plan. Record account numbers and contact information. Plan with trusted individuals what should be done if there is an emergency need for more money. Understand ahead of time the best way to carry money, how you can obtain funds from your accounts, how other people can send you money, and how funds are best handled (e.g., cash, credit cards, ATM) at your destination. Keeping a small amount of cash in your emergency kit make the difference in survival in some countries and situations.

5. Examine the weather records. One of the few things we can count on it that weather changes. Keep up on reports as often as is reasonably possible. In some cases, it is appropriate to carry a short-wave or other radio that allows periodic checks of local and regional weather.

6. Identify the most likely health concerns and how you will handle them. For example, if you anticipate problems with chiggers or poisonous snakes, what are the ways to prevent problems and, if they occur, what steps should be taken to minimize problems? Check your list with trusted world-wide web information and then confirm you findings with appropriate professionals (such as your doctor).

7. Transport yourself and your equipment defensively. It is very easy to loose important equipment through poor transportation habits. Store equipment in multiple sealed containers (sealing plastic storage bags are very useful). Use dry bags and reinforced bags that are unlikely to tear or allow contents to become wet. This is particularly important for emergency kits. Heavy straps around luggage that might open will help keep contents intact.

8. Establish a communication protocol. You should be in regular contact with people so that they know if you are likely to be in trouble. This operates at several scales. Daily information about your travel, communication events that if missed will signal that there is something wrong. The same individuals who can serve to trigger an emergency notice if you fail to report in should have complete information about your safety equipment and skills that will both reassure them and be of assistance to potential rescuers.

9. Maintain a cache of food and water. Many situations may prevent you from obtaining food and water in normal ways, such as power outages, holidays, extreme weather, illness, and transportation strikes. A small store of key items (such as peanut butter) can help you through the difficult period.

10. Build, carry and know how to use your emergency field kits. One of the most foolish things to do is buy a fancy emergency kit and then never learn to use it until an emergency happens. Try the kit out, make sure that everything in it is useful and that you are clear on how to use it (not just theoretically, but from experience through practice.) 